\title{
Meta-analysis and systematic review of risk factors for shunt dependency after aneurysmal subarachnoid hemorrhage
}

\author{
${ }^{*}$ Christopher D. Wilson, BS, ${ }^{1}$ Sam Safavi-Abbasi, PhD, ${ }^{2}$ Hai Sun, MD, PhD, ${ }^{2}$ \\ M. Yashar S. Kalani, PhD, ${ }^{2}$ Yan D. Zhao, PhD, ${ }^{3}$ Michael R. Levitt, MD, ${ }^{2}$ \\ Ricardo A. Hanel, MD, PhD, ${ }^{4}$ Eric Sauvageau, MD, ${ }^{4}$ Timothy B. Mapstone, MD, ${ }^{1}$ \\ Felipe C. Albuquerque, MD, ${ }^{2}$ Cameron G. McDougall, MD, ${ }^{2}$ Peter Nakaji, MD, ${ }^{2}$ and \\ Robert F. Spetzler, MD²
}

\begin{abstract}
Departments of ${ }^{1}$ Neurosurgery and ${ }^{3}$ Biostatistics and Epidemiology, University of Oklahoma, Oklahoma City, Oklahoma; 2Department of Neurosurgery, Barrow Neurological Institute, St. Joseph's Hospital and Medical Center, Phoenix, Arizona; and ${ }^{4}$ Lyerly Neurosurgery, Baptist Hospital, Jacksonville, Florida
\end{abstract}

\begin{abstract}
OBJECTIVE Aneurysmal subarachnoid hemorrhage (aSAH) may be complicated by hydrocephalus in $6.5 \%-67 \%$ of cases. Some patients with aSAH develop shunt dependency, which is often managed by ventriculoperitoneal shunt placement. The objectives of this study were to review published risk factors for shunt dependency in patients with aSAH, determine the level of evidence for each factor, and calculate the magnitude of each risk factor to better guide patient management.
\end{abstract}

METHODS The authors searched PubMed and MEDLINE databases for Level A and Level B articles published through December 31, 2014, that describe factors affecting shunt dependency after aSAH and performed a systematic review and meta-analysis, stratifying the existing data according to level of evidence.

RESULTS On the basis of the results of the meta-analysis, risk factors for shunt dependency included high Fisher grade (OR 7.74, 95\% Cl 4.47-13.41), acute hydrocephalus (OR 5.67, 95\% Cl 3.96-8.12), in-hospital complications (OR 4.91, $95 \% \mathrm{Cl} 2.79-8.64)$, presence of intraventricular blood (OR 3.93, 95\% Cl 2.80-5.52), high Hunt and Hess Scale score (OR 3.25, 95\% Cl 2.51-4.21), rehemorrhage (OR 2.21, 95\% Cl 1.24-3.95), posterior circulation location of the aneurysm (OR 1.85, 95\% Cl 1.35-2.53), and age $\geq 60$ years (OR 1.81, 95\% Cl 1.50-2.19). The only risk factor included in the meta-analysis that did not reach statistical significance was female sex (OR 1.13, 95\% Cl 0.77-1.65).

CONCLUSIONS The authors identified several risk factors for shunt dependency in aSAH patients that help predict which patients are likely to require a permanent shunt. Although some of these risk factors are not independent of each other, this information assists clinicians in identifying at-risk patients and managing their treatment.

http://thejns.org/doi/abs/10.3171/2015.11.JNS152094

KEY WORDS aneurysmal subarachnoid hemorrhage; cerebral aneurysm rupture; Fisher grade; hydrocephalus; risk factors; shunt dependency; vascular disorders

$\mathrm{H}$ YDROCEPHALUS generally complicates $20 \%-35 \%$ of aneurysmal subarachnoid hemorrhages (aSAHs). ${ }^{27}$ However, reported frequencies range from $6.5 \%$ to $67 \%{ }^{14,16,19,27}$ Hydrocephalus increases morbidity ${ }^{14,16,18,19,22}$ and confers longer hospital and intensive care unit stays. ${ }^{18}$ External ventricular drain (EVD) placement is usually required for acute management of hydrocephalus, but because of infection risk, EVDs are temporary and must eventually be removed; however, $8 \%-63 \%$ of these patients require permanent CSF diversion, usually with ventriculoperitoneal shunting. ${ }^{17}$

Since the association between aSAH and hydrocephalus was described in 1928, the occurrence of hydrocephalus after aSAH has been well studied, but reports of risk factors for its development are inconsistent..$^{13}$ Therefore, factors that predict shunt dependency are not clear, and

ABBREVIATIONS aSAH = aneurysmal subarachnoid hemorrhage; $\mathrm{BCI}=$ bicaudate index; $\mathrm{EVD}=$ external ventricular drain.

SUBMITTED September 4, 2015. ACCEPTED November 3, 2015.

INCLUDE WHEN CITING Published online April 1, 2016; DOI: 10.3171/2015.11.JNS152094.

* Mr. Wilson and Dr. Safavi-Abbasi contributed equally to this work. 
hydrocephalus after aSAH is still a significant hazard to patients. The goals of our review were 3 -fold. First, we systematically reviewed the literature to identify all published risk factors for shunt dependency in aSAH patients. Second, we combined data to calculate the magnitude of each risk factor. Third, we organized the literature according to the level of evidence. Our work will assist practitioners as they manage patients with aSAH by providing evidence-based risk factors for shunt dependency.

\section{Methods}

\section{Search Strategy and Selection Criteria}

We reviewed PubMed and MEDLINE for articles pertaining to aSAH, hydrocephalus, and shunt dependency, published by December 31, 2014. We searched titles and abstracts for the terms "aneurysmal subarachnoid hemorrhage" and "shunt dependency" combined with the following: ventriculoperitoneal shunt, bicaudate index, shunt-dependency risk factors, neurological grade, Glasgow Coma Scale score, Hunt and Hess Scale score, Fisher grade, World Federation of Neurosurgical Societies grade, sex, and age. We used no other search or language restrictions. Identified studies were screened independently by title and abstract by 2 reviewers (C.D.W. and S.S.A.). If articles could not initially be included or excluded, their full text was evaluated. The reference lists of relevant articles were manually searched to ensure that no published data were missed. The lists of included studies generated by each author were compared, and any discrepancies were resolved by consensus.

\section{Study Selection and Data Extraction}

The quality of the body of evidence evaluating each risk factor was assessed using the GRADE (Grading of Recommendations, Assessment, Development, and Evaluation) system; evidence was stratified as Level A, B, or C, according to American Heart Association Stroke Counsel methods. ${ }^{1,6}$ No studies were excluded due to bias, but the possible sources of bias are explained below. Level A or B studies that discussed the effect of at least 1 risk factor on shunt dependency were included. Data extraction from each study was performed independently by C.D.W. and was then evaluated for accuracy by S.S.A. Results for each risk factor are described by level of evidence in the following text, tables, and figures, with the most recent publications listed first. Any patient-related risk factor that was evaluated by at least 2 studies that provided extractable data for OR calculation were evaluated by meta-analysis.

\section{Statistical Analysis}

For each risk factor, we combined data into an overriding OR and 95\% CI using meta-analysis. All articles with extractable data for OR calculation were used. Data were extracted from tables and from the associated text describing the incidence of each risk factor and shunt dependency. The homogeneity of ORs was tested using Cochran's Q statistics. ${ }^{4}$ If homogeneity was rejected at the 0.1 level, then ORs were treated as random effects, and the overall OR was computed using the Cochran-Mantel-Haenszel method $^{5}$; otherwise, ORs were treated as fixed effects, and overall ORs were computed using the DerSimonian-Laird method. ${ }^{10}$ We assessed for publication bias by calculating the standard errors of the ORs from each study and constructing funnel plots for each risk factor. If data were not presented in a way that allowed OR calculation, the article was included for evidence stratification.

\section{Role of the Funding Source}

There was no funding source for this study. All authors had full access to all data used in the study, and C.D.W., S.S.A., and R.F.S. were responsible for the decision to submit the study for publication.

\section{Results}

We identified 75 unduplicated articles electronically and by manual search. Of these, we eliminated 32 with Level C evidence, 10 that reported on brain pathology other than aSAH, and 11 that were deemed irrelevant. Twenty-two articles were ultimately included, and 21 of these provided data extractable for meta-analysis (21 Level B; Fig. 1). Evidence for and against each risk factor is stratified in Table 1.

\section{Fisher Grade}

High Fisher grade ( 3 or 4 ) was evaluated in 10 articles. All provided Level B evidence. ${ }^{7-9,11,13,15,19,22,27,28}$ High Fisher grade was the strongest risk factor for shunt dependency in our meta-analysis. Fisher grades 3 or 4 conferred a nearly 8 -fold increase in shunt dependency compared with Fisher grades 1 or 2 (Fig. 2; OR 7.74, 95\% CI 4.47-13.41). Considered a whole, the body of evidence that supports this conclusion is high grade according to the GRADE system. ${ }^{1}$ All but $1^{27}$ of the 10 studies showed significant increases in shunt dependency in patients with high Fisher grades. ${ }^{8,9,11,13,15,19,22,28}$ The 1 study that did not show significant increases involved a small cohort, only 120 patients, which may explain its nonsignificance; however, other studies involving fewer patients demonstrated a significant association between this risk factor and shunt dependency. Included data regarding high Fisher grade may be subject to publication bias (Supplemental Fig. 1).

\section{Acute Hydrocephalus}

Acute hydrocephalus was evaluated in 11 studies. All

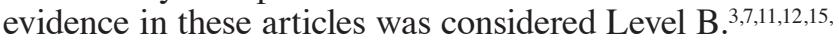
18-20,23,27,29 In our meta-analysis, acute hydrocephalus conferred a nearly 6-fold increase in odds of shunt dependency (Fig. 3; OR 5.67, 95\% CI 3.96-8.12). The body of evidence for this risk factor was considered high grade. ${ }^{1}$ All studies demonstrated significant increases in shunt dependency in patients with this risk. $3,7,11,12,15,18-20,27,29$ Funnel plot analysis for acute hydrocephalus data indicated no concern for publication bias (Supplemental Fig. 2).

\section{In-Hospital Complications}

In-hospital complications associated with shunt dependency were evaluated in 11 Level B studies. ${ }^{2,3,7,11,12,15,16,19-21,24}$ The in-hospital complications included were nosocomial meningitis, pneumonia, vasospasm, and ischemic stroke., ${ }^{711,12,15,16,19-21}$ All were significant risk factors in at 


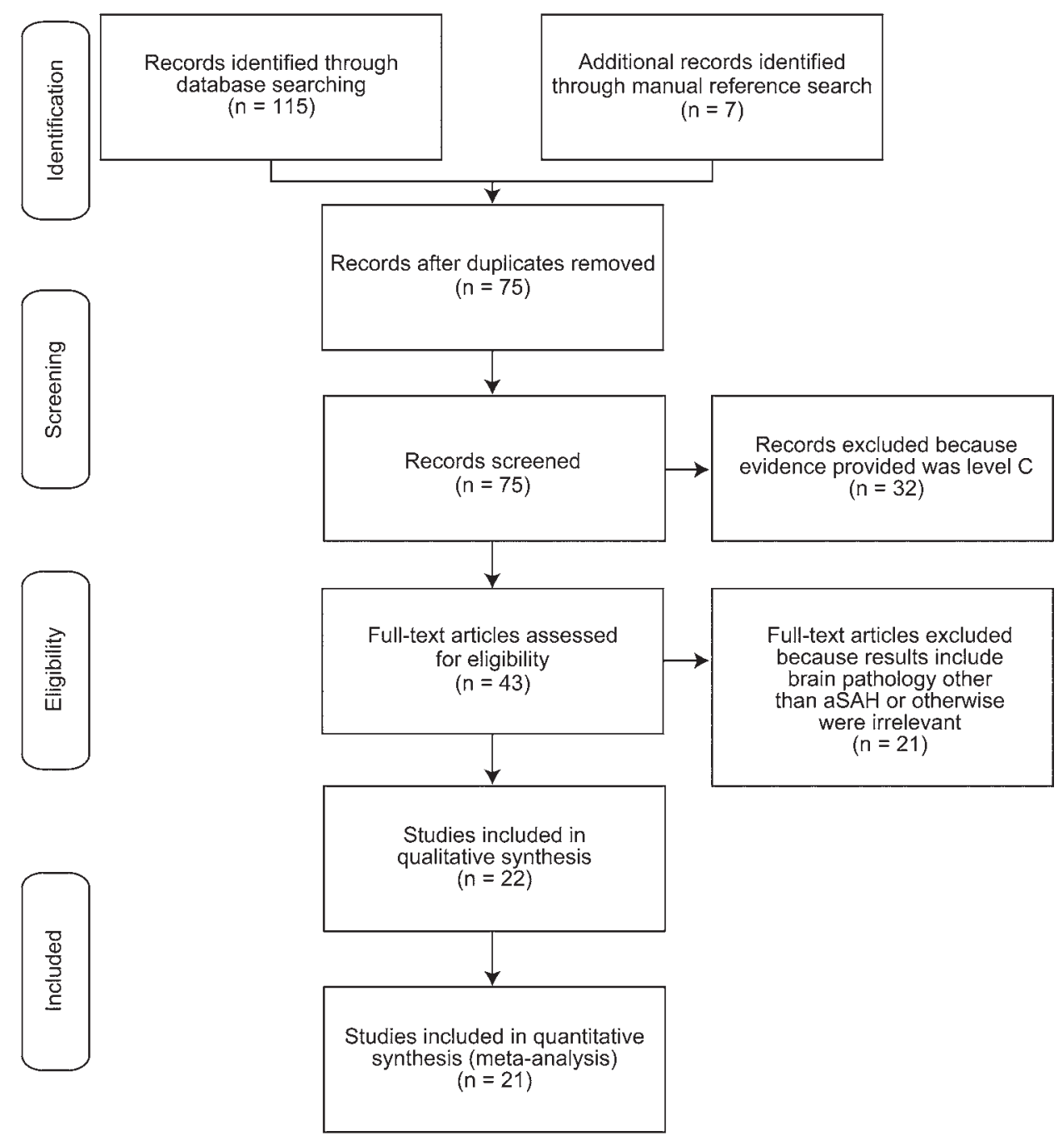

FIG. 1. Preferred Reporting Items for Systematic Reviews and Meta-Analysis (PRISMA) flow chart of scientific literature search and study selection.

least 1 published study. According to our meta-analysis, aSAH patients with any of these complications during hospitalization carried a nearly 5-fold higher shunt-dependency risk than patients without these complications (Fig. 4; OR 4.91, 95\% CI 2.79-8.64). The body of evidence included for this analysis was considered high grade. ${ }^{1}$ Despite our collective examination of different complications, variation among studies was small, and all studies revealed a significant increase in shunt dependency in patients with these complications. When the factors were analyzed independently, nosocomial meningitis conferred the highest risk, followed by ischemic stroke, clinical vasospasm, and, finally, pneumonia, according to Level B evidence. ${ }^{11,20}$ Mixed evidence supported vasospasm and ischemic stroke..$^{12,15,19,24}$ There was no concern for publication bias within the in-hospital complication data (Supplemental Fig. 3).

\section{Intraventricular Blood}

The association of intraventricular blood with shunt dependency was evaluated in 13 Level B studies. ${ }^{11-13,15,16,19-25,27}$
Our meta-analysis indicated that the presence of intraventricular blood in aSAH patients conferred a nearly 4-fold increased shunt-dependency risk compared with that in patients without intraventricular blood (Fig. 5; OR 3.93, 95\% CI 2.80-5.52). The body of evidence regarding intraventricular blood was considered high grade. ${ }^{1}$ Yamada et al. ${ }^{27}$ found the highest odds of shunt dependency in patients with intraventricular blood (OR 13.60, 95\% CI 5.1336.06). However, this study was characterized by extremely long hospital stays, which may have exposed patients to in-hospital complications, which are risk factors themselves. Two studies demonstrated wide CIs secondary to small sample sizes. ${ }^{25,27}$ However, all studies ${ }^{11-13,15,16,19-25,27}$ included in our meta-analysis demonstrated statistical significance. Fourth ventricle blood conferred the highest shunt-dependency risk, followed by lateral ventricle hemorrhage, and then third ventricle hemorrhage. ${ }^{19}$ Shunt dependency was also directly proportional to the thickness of blood, similar to high Fisher grade. ${ }^{11,12}$ Intraventricular blood data may be subject to publication bias (Supplemental Fig. 4). 
TABLE 1. Literature summary of major risk factors for shunt dependency*

\begin{tabular}{|c|c|c|c|}
\hline Risk Factor & Supporting Evidence & Refuting Evidence & $\begin{array}{l}\text { Meta-Analysis } \\
\text { OR }(95 \% \mathrm{Cl})\end{array}$ \\
\hline High Fisher grade & $\begin{array}{l}\text { Yang et al., 2013; Rincon et al., 2010; Kwon et al., 2008; de } \\
\text { Oliveira et al., 2007; Dehdashti et al., 2004; Dorai et al., } \\
\text { 2003; Demirgil et al., 2003; Gruber et al., 1999; Vale et al., } \\
1997\end{array}$ & Yamada et al., 2012 & $7.74(4.47-13.41)$ \\
\hline $\begin{array}{l}\text { AH at admissiont } \\
\& \text { EVD chal- } \\
\text { lenge }\end{array}$ & $\begin{array}{l}\text { Yu et al., 2014; Yamada et al., 2012; Rincon et al., 2010; de } \\
\text { Oliveira et al., 2007; O'Kelly et al., 2009; Chan et al., 2009; } \\
\text { Kwon et al., 2008; Dorai et al., 2003; Sheehan et al., 1999; } \\
\text { Graff-Radford et al., 1989; van Gijn et al., } 1985\end{array}$ & None & $5.67(3.96-8.12)$ \\
\hline $\mathrm{IHC} \ddagger$ & $\begin{array}{l}\text { Lai \& Morgan, 2013; Brandner et al., 2012; Rincon et al., } \\
\text { 2010; Chan et al., 2009; Kwon et al., 2008§; de Oliveira et } \\
\text { al., 2007; Dorai et al., 2003; Sheehan et al., 1999; Tapani- } \\
\text { naho et al., 1993; Graff-Radford et al., } 1989\end{array}$ & $\begin{array}{l}\text { Rincon et al., 2010; Kwon et al., 2008§; Vermeij } \\
\quad \text { et al., } 1994\end{array}$ & $4.91(2.79-8.64)$ \\
\hline IVB & $\begin{array}{l}\text { Lai \& Morgan, 2013; Wang et al., 2012; Yamada et al., 2012; } \\
\quad \text { Rincon et al., 2010; Kwon et al., 2008; Dorai et al., 2003; } \\
\text { Gruber et al., 1999; Vale et al., 1997; Vermeij et al., 1994; } \\
\text { Tapaninaho et al., 1993; Sheehan et al., 1999; Graff- } \\
\text { Radford et al., 1989; van Gijn et al., } 1985\end{array}$ & None & $3.93(2.80-5.52)$ \\
\hline $\begin{array}{l}\text { High HHS score } \\
\text { at admission }\end{array}$ & $\begin{array}{l}\text { Yu et al., 2014; Yang et al., 2013; Brandner et al., 2012; } \\
\text { Rincon et al., 2010; O'Kelly et al., 2009; Chan et al., 2009; } \\
\text { Kwon et al., 2008; de Oliveira et al., 2007; Dorai et al., } \\
\text { 2003; Gruber et al., 1999; Vale et al., 1997; Graff-Radford } \\
\text { et al., } 1989\end{array}$ & Demirgil et al., 2003 & $3.25(2.51-4.21)$ \\
\hline $\mathrm{RH}$ & Gruber et al., 1999 & Rincon et al., 2010 & $2.21(1.24-3.95)$ \\
\hline PC & $\begin{array}{l}\text { Yu et al., 2014; O’Kelly et al., 2009; Chan et al., 2009; Dorai } \\
\text { et al., 2003; Sheehan et al., 1999; Tapaninaho et al., 1993; } \\
\text { Graff-Radford et al., } 1989\end{array}$ & $\begin{array}{l}\text { Lai \& Morgan, 2013; Woernle et al., 2013; Yang } \\
\text { et al., 2013; Yamada et al., 2012; Kwon et al., } \\
\text { 2008; Gruber et al., } 1999\end{array}$ & $1.85(1.35-2.53)$ \\
\hline Age $\geq 60$ yrs & $\begin{array}{l}\text { Yu et al., 2014; Yamada et al., 2012; O'Kelly et al., 2009; } \\
\text { Kwon et al., 2008; Dorai et al., 2003; Tapaninaho et al., } \\
\text { 1993; Graff-Radford et al., } 1989\end{array}$ & Sheehan et al., 1999; Vale et al., 1997 & $1.81(1.50-2.19)$ \\
\hline Female sex & Chan et al., 2009; Dorai et al., 2003 & $\begin{array}{l}\text { Yu et al., 2014; Woernle et al., 2013; Yang et } \\
\text { al., 2013; Brandner et al., 2012; Rincon et al., } \\
\text { 2010; Kwon et al., 2008; de Oliveira et al., } \\
\text { 2007; Sheehan et al., 1999; Vale et al., 1997; } \\
\text { Vermeij et al., 1994; Tapaninaho et al., } 1993\end{array}$ & $1.13(0.77-1.65)$ \\
\hline
\end{tabular}

$\mathrm{AH}=$ acute hydrocephalus; $\mathrm{HHS}=$ Hunt and Hess Scale; IHC = in-hospital complications; IVB = intraventricular blood; $\mathrm{PC}=$ posterior circulation aneurysm location; RH $=$ rehemorrhage.

* All evidence is Level $B$.

$\dagger$ Increased $\mathrm{BCl}$ and large third ventricular diameter.

$\ddagger$ Nosocomial meningitis, pneumonia, vasospasm, and ischemic stroke.

$\S$ Kwon et al. reported a significant increase in shunt dependency if nosocomial meningitis developed but not if vasospasm or ischemic stroke developed.

\section{Hunt and Hess Scale}

The association of high Hunt and Hess Scale scores (3-5) with shunt dependency was evaluated in 13 Level B studies. . $3,7,9,11-13,15,18,19,22,28,29$ Eleven studies ${ }^{2,7,9,11-13,15,19,22,28,29}$ were included in our meta-analysis, which demonstrated more than 3-fold increased shunt-dependency risk with high Hunt and Hess Scale scores compared with lower scores (1 or 2) (Fig. 6; OR 3.25, 95\% CI 2.51-4.21). The body of evidence supporting this risk factor was considered high grade. ${ }^{1}$ Only 1 small study, which had a wide CI, showed no significant increase in shunt dependency with high scores (OR 2.00, 95\% CI 0.86-4.68). ${ }^{9}$ Larger studies with similar ORs showed statistical significance. ${ }^{12,15,19}$ There is no indication of publication bias regarding high Hunt and Hess Scale data (Supplemental Fig. 5).

\section{Rehemorrhage}

The association between rehemorrhage and shunt dependency was evaluated in 2 Level B studies. ${ }^{13,19}$ Both were included in our meta-analysis, which demonstrated more than a 2-fold increased odds of shunt dependency in patients with rehemorrhage after initial aSAH compared with patients without rehemorrhage (Fig. 7; OR $2.21,95 \%$ CI 1.24-3.95). The body of evidence supporting this risk factor was considered very low grade. ${ }^{1}$ Although our analysis showed a significant OR, 1 of the 2 studies did not show a significant increase in shunt dependency after rehemorrhage ${ }^{19}$ whereas the other study did note a significant increase. ${ }^{13}$ Publication bias is difficult to assess because only 2 studies were included; however, the associated funnel plot was symmetric (Supplemental Fig. 6). 


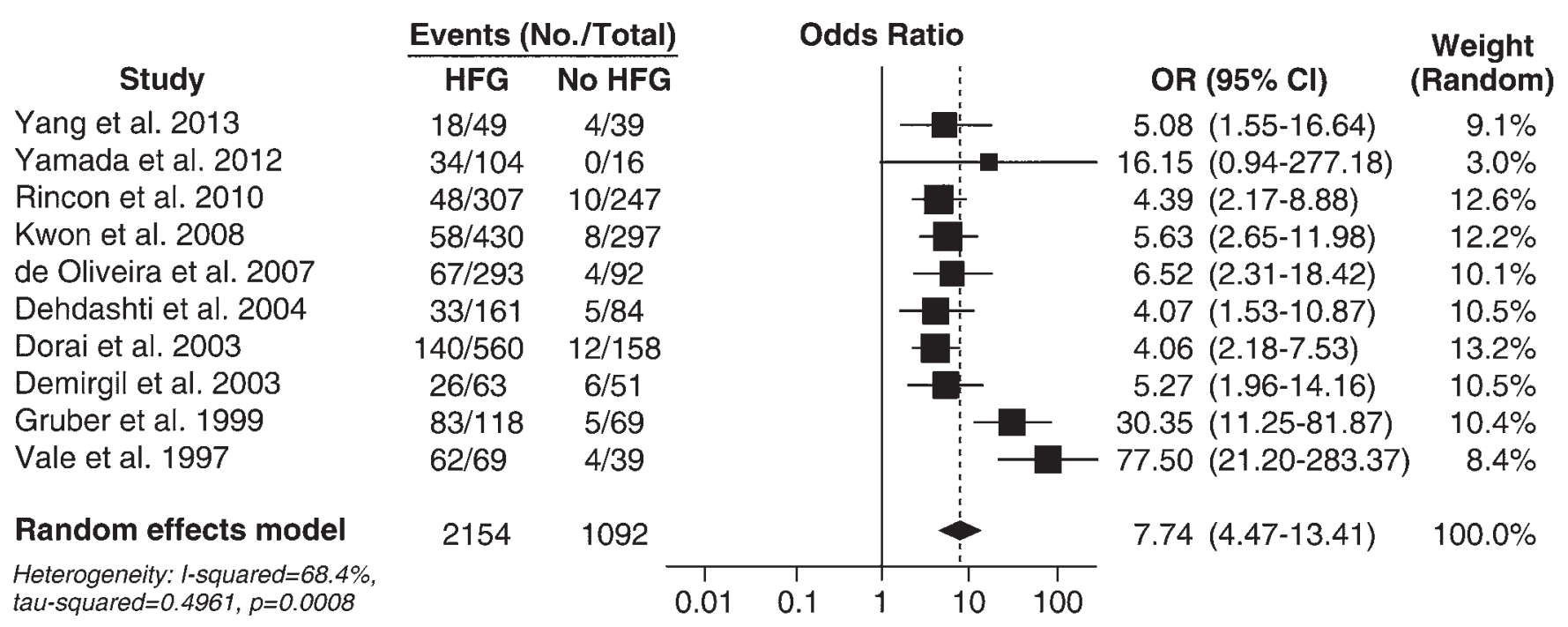

FIG. 2. Forest plot for high Fisher grade (HFG, defined as Fisher grades 3-4) as a risk factor for shunt dependency compared with low Fisher grade (No HFG, defined as Fisher grades 1-2).

\section{Posterior Circulation Aneurysm Location}

The association of posterior circulation aneurysm location (defined as aneurysms arising from the vertebral, basilar, or posterior communicating arteries or their branches) with shunt dependency was evaluated in 13 Level B studies. ${ }^{3,11-13,15,16,18,20,21,26-29}$ Our meta-analysis included 11 of these studies, , 311,12,15,16,18,21,26-29 and we found that having a posterior circulation aneurysm increased the risk of shunt dependency nearly 2 -fold compared with patients who had aneurysms elsewhere (Fig. 8; OR 1.85, 95\% CI 1.35-2.53). Patients with multiple aneurysms were excluded from the analysis. The body of evidence included in the evaluation of this risk factor was considered moderate grade. ${ }^{1}$ Posterior circulation aneurysm location was one of the strongest predictors of shunt dependency in the early cohort of Tapaninaho et al. ${ }^{21}$ However, several newer studies demonstrate no association of posterior aneurysms with shunt dependency; some results have wide CIs, which may explain their lack of statistical significance. . $^{15,16,26-28}$ There was no indication of publication bias regarding the posterior circulation aneurysm data (Supplemental Fig. 7).

\section{Older Age}

The association of increased age with shunt dependency was evaluated in 9 Level B articles. . $^{11,12,15,18,20-22,27,29}$ Only 2 studies presented extractable data for inclusion in our meta-analysis, ${ }^{12,15}$ which indicated that aSAH patients aged 60 years and older had a nearly 2 -fold increase in the rate of shunt dependency compared with younger patients (Fig. 9; OR 1.81, 95\% CI 1.50-2.19). Other studies report similar results..$^{11,18,20-22,27}$ The body of evidence supporting older age as a risk factor for shunt dependency was considered moderate grade. ${ }^{1}$ We chose 60 years of age as our cutoff between young and old cohorts because shunt dependency increases significantly at age 60 years, on the basis of the sum of the incidences in both studies included in our meta-analysis for ages above and below 60 years. ${ }^{16,20}$ We found the overall incidence of shunt dependency in patients aged 60-69 years and in patients aged 70 years and older to be $19.5 \%$ and $30.5 \%$, respectively. ${ }^{12,15}$ In comparison, aSAH patients younger than 60 years had an $11.3 \%$ incidence of shunt dependency $(\mathrm{p}<0.0001$ relative to patients $\geq 60$ years). ${ }^{12,15}$ No indication of publication

\begin{tabular}{|c|c|c|c|c|c|}
\hline \multirow[b]{2}{*}{ Study } & \multicolumn{2}{|c|}{ Events (No./Total) } & \multicolumn{2}{|l|}{ Odds Ratio } & \multirow{2}{*}{$\begin{array}{l}\text { Weight } \\
\text { (Random) }\end{array}$} \\
\hline & $\mathrm{AH}$ & No AH & & OR $(95 \% \mathrm{Cl})$ & \\
\hline Yu et al. 2014 & $25 / 48$ & $40 / 202$ & & $4.40(2.27-8.55)$ & $12.3 \%$ \\
\hline Yamada et al. 2012 & $19 / 34$ & $15 / 86$ & & $6.00(2.50-14.41)$ & $9.4 \%$ \\
\hline O’Kelly et al. 2009 & $103 / 227$ & $482 / 2893$ & & $4.15(3.14-5.49)$ & $18.9 \%$ \\
\hline Kwon et al. 2008 & $43 / 156$ & $23 / 578$ & & $9.18(5.32-15.84)$ & $14.3 \%$ \\
\hline de Oliveira et al. 2007 & $61 / 205$ & $10 / 180$ & & $7.20(3.56-14.57)$ & $11.7 \%$ \\
\hline Dorai et al. 2003 & $105 / 338$ & $47 / 380$ & & $3.19(2.18-4.68)$ & $17.2 \%$ \\
\hline Sheehan et al. 1999 & $116 / 292$ & $29 / 456$ & & $9.70(6.23-15.12)$ & $16.1 \%$ \\
\hline Random effects model & 1300 & 4775 & & $5.67(3.96-8.12)$ & $100.0 \%$ \\
\hline $\begin{array}{l}\text { Heterogeneity: } 1 \text {-squared }=72.3 \% \\
\text { tau-squared }=0.157, p=0.0014\end{array}$ & & & .051 & & \\
\hline
\end{tabular}

FIG. 3. Forest plot for acute hydrocephalus $(\mathrm{AH})$ as a risk factor for shunt dependency compared with no hydrocephalus at presentation. Acute hydrocephalus was defined as ventriculomegaly on initial brain CT imaging. 


\begin{tabular}{|c|c|c|c|c|c|}
\hline \multirow[b]{2}{*}{ Study } & \multicolumn{2}{|c|}{ Events (No./Total) } & \multicolumn{2}{|l|}{ Odds Ratio } & \multirow{2}{*}{$\begin{array}{l}\text { Weight } \\
\text { (Random) }\end{array}$} \\
\hline & IC & No IC & & OR $(95 \%$ Cl) & \\
\hline Lai and Morgan 2013 & $43 / 134$ & $658 / 10,673$ & & $7.19(4.96-10.42)$ & $21.9 \%$ \\
\hline Rincon et al. 2010 & $10 / 28$ & $51 / 546$ & & $5.39(2.36-12.30)$ & $16.0 \%$ \\
\hline Kwon et al. 2008 & $7 / 13$ & $59 / 721$ & & $13.09(4.26-40.22)$ & $12.4 \%$ \\
\hline de Oliveira et al. 2007 & $33 / 132$ & $11 / 100$ & & $2.70(1.29-5.65)$ & $17.1 \%$ \\
\hline Dorai et al. 2003 & $36 / 102$ & $116 / 616$ & & $2.35(1.49-3.70)$ & $20.9 \%$ \\
\hline Sheehan et al. 1999 & $9 / 13$ & $161 / 643$ & & $6.74(2.05-22.17)$ & $11.7 \%$ \\
\hline Random effects model & 422 & 13,299 & & $4.91(2.79-8.64)$ & $100.0 \%$ \\
\hline $\begin{array}{l}\text { Heterogeneity: } 1 \text {-squared }=76 \% \text {, } \\
\text { tau-squared }=0.3438, p=0.0009\end{array}$ & & 0.02 & $\begin{array}{lll}1 & 1 & 1 \\
0.51 & 2\end{array}$ & & \\
\hline
\end{tabular}

FIG. 4. Forest plot for in-hospital complications (IC) as a risk factor for shunt dependency compared with patients with no complications during their hospital stay. Complications included were nosocomial meningitis, pneumonia, vasospasm, and ischemic stroke.

bias was identified on funnel plot analysis (Supplemental Fig. 8).

\section{Female Sex}

The association between female sex and shunt dependency was evaluated in 13 Level B studies. ${ }^{2,3,7,11,15,19-22,}$ 24,26,28,29 Published results were mixed, and our metaanalysis showed no significant increase in shunt dependency in female aSAH patients (Fig. 10; OR 1.13, 95\% CI 0.77-1.65). The body of evidence used to evaluate female sex regarding shunt dependency was considered moderate grade. ${ }^{1}$ Two studies showed a nonsignificant increase in shunt dependency in male patients. ${ }^{7,28}$ One Level B study demonstrated significantly increased shunt dependency in males. ${ }^{20}$ Only 2 studies showed a significant increase in shunt dependency in female patients. ${ }^{3,11}$ All others showed nonsignificantly increased shunt dependency in females. ${ }^{2,15,19,21,22,24,26,29}$ We found no indication of publication bias for data regarding this risk factor (Supplemental Fig. 9).

\section{Discussion}

High Fisher grade was the strongest predictor of shunt dependency among aSAH patients in our meta-analysis. All but 1 small study reported a statistically significant difference for this factor. The outlier (OR 16.15, 95\% CI 0.94-277.18) had a wide CI and a small sample, with only 16 of 120 patients having low Fisher grades. None of those with low Fisher grades became shunt dependent. ${ }^{27}$ One Level B study indicated a particularly large effect (OR 30.35 , 95\% CI 11.25-81.87). ${ }^{13}$ In this study, ${ }^{13}$ a preponderance of patients underwent aneurysm clipping, which has been associated with shunt dependency. ${ }^{29}$ Vale et al. ${ }^{22}$ demonstrated the highest odds of shunt dependency in patients with high Fisher grades (OR 77.50, 95\% CI 21.20283.37). They suggest that this extraordinarily high OR is a consequence of their study occurring at a major referral center that receives complicated patients from smaller centers. However, other studies that had smaller ORs were also derived from large referral centers. The relationship between high Fisher grade and shunt dependency can likely be explained because high Fisher grade can be caused by increased arachnoid villi scarring and occlusion when there is more blood. 27

Acute hydrocephalus was the second largest risk factor in our meta-analysis. Any ventriculomegaly on initial imaging may be indicative of acute hydrocephalus., ${ }^{3,27}$ How-

\begin{tabular}{|c|c|c|c|c|c|}
\hline \multirow[b]{2}{*}{ Study } & \multicolumn{2}{|c|}{ Events (No./Total) } & Odds Ratio & \multirow[b]{2}{*}{ OR $(95 \% \mathrm{Cl})$} & \multirow{2}{*}{$\begin{array}{c}\text { Weight } \\
\text { (Random) }\end{array}$} \\
\hline & IB & No IB & & & \\
\hline Lai and Morgan 2013 & $43 / 288$ & $658 / 10,519$ & & $2.63(1.88-3.67)$ & $19.6 \%$ \\
\hline Yamada et al. 2012 & $27 / 46$ & $7 / 74$ & & $13.60(5.13-36.06)$ & $8.1 \%$ \\
\hline Wang et al. 2012 & $6 / 11$ & $9 / 75$ & & $8.80(2.22-34.84)$ & $4.9 \%$ \\
\hline Rincon et al. 2010 & $41 / 61$ & $184 / 513$ & & $3.67(2.08-6.44)$ & $14.5 \%$ \\
\hline Kwon et al. 2008 & $34 / 136$ & $32 / 598$ & & $5.90(3.48-9.98)$ & $15.3 \%$ \\
\hline Dorai et al. 2003 & $81 / 253$ & $71 / 465$ & & $2.61(1.81-3.77)$ & $18.9 \%$ \\
\hline Sheehan et al. 1999 & $110 / 287$ & $59 / 367$ & & $3.24(2.25-4.68)$ & $18.9 \%$ \\
\hline Random effects model & 1082 & 12,611 & & $3.93(2.80-5.52)$ & $100.0 \%$ \\
\hline $\begin{array}{l}\text { Heterogeneity: } 1 \text {-squared }=67 \% \text {, } \\
\text { tau-squared }=0.1243, p=0.0058\end{array}$ & & 0.03 & 0.512 & & \\
\hline
\end{tabular}

FIG. 5. Forest plot for the presence of intraventricular blood (IB) as a risk factor for shunt dependency compared with patients with no intraventricular blood on presentation. 


\begin{tabular}{|c|c|c|c|c|c|}
\hline \multirow[b]{2}{*}{ Study } & \multicolumn{2}{|c|}{ Events (No./Total) } & \multicolumn{2}{|l|}{ Odds Ratio } & \multirow{2}{*}{$\begin{array}{l}\text { Weight } \\
\text { (Random) }\end{array}$} \\
\hline & High HHS & Low HHS & & OR (95\% Cl) & \\
\hline Yu et al. 2014 & $23 / 58$ & $17 / 144$ & & $4.91(2.37-10.19)$ & $8.2 \%$ \\
\hline Yang et al. 2013 & $12 / 29$ & $10 / 59$ & & $3.46(1.27-9.44)$ & $5.2 \%$ \\
\hline Brandner et al. 2012 & $22 / 38$ & $3 / 30$ & & $12.38(3.19-48.00)$ & $3.2 \%$ \\
\hline Rincon et al. 2010 & $44 / 317$ & $17 / 263$ & 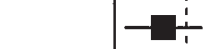 & $2.33(1.30-4.19)$ & $10.7 \%$ \\
\hline Kwon et al. 2008 & $32 / 251$ & $34 / 483$ & & $1.93(1.16-3.21)$ & $12.4 \%$ \\
\hline de Oliveira et al. 2007 & $56 / 211$ & $15 / 174$ & & $3.83(2.08-7.06)$ & $10.2 \%$ \\
\hline Dorai et al. 2003 & $123 / 402$ & $29 / 316$ & & $4.36(2.82-6.75)$ & $14.1 \%$ \\
\hline Demirgil et al. 2003 & $21 / 61$ & $11 / 53$ & & $2.00(0.86-4.68)$ & $6.7 \%$ \\
\hline Gruber et al. 1999 & $33 / 110$ & $7 / 77$ & & $4.29(1.78-10.31)$ & $6.4 \%$ \\
\hline Vale et al. 1997 & $17 / 60$ & $2 / 48$ & & $9.09(1.98-41.70)$ & $2.6 \%$ \\
\hline Graff-Radford et al. 1989 & $328 / 1799$ & $138 / 1722$ & E & $2.56(2.07-3.16)$ & $20.4 \%$ \\
\hline Random effects model & 3336 & 3369 & & $3.25(2.51-4.21)$ & $100.0 \%$ \\
\hline $\begin{array}{l}\text { Heterogeneity: } 1 \text {-squared }=46.5 \%, \\
\text { tau-squared }=0.074, p=0.0443\end{array}$ & & 0.02 & $\begin{array}{llll} & & & \\
0.5 & 1 & 2\end{array}$ & & \\
\hline
\end{tabular}

FIG. 6. Forest plot for high Hunt and Hess Scale scores (High HHS, defined as a score of 3-5) as a risk factor for shunt dependency compared with patients with low scores (Low HHS, defined as a score of 1-2).

ever, calculation of the bicaudate index (BCI) on neuroimaging is used for objective diagnosis., $32,15,18,20,27$ The BCI is well described by van Gijn et al. ${ }^{23}$ Briefly, the distance between the lateral edges of the frontal horns at the level of the caudate nuclei is divided by the diameter of the brain at the same level. ${ }^{23}$ There is considerable variation regarding the upper limits of a normal BCI, which increases with aging due to brain atrophy. $3,12,15,18,20,27$ However, many studies $^{3,12,15,16,18,20,27}$ have indicated shunt dependency was more common in patients with $\mathrm{BCI} \geq 0.20$.

Four in-hospital complications were associated with shunt dependency: meningitis, pneumonia, vasospasm, and ischemic stroke. Although these complications are distinct entities, there was not enough evidence to analyze each individually. Therefore, we considered these 4 most-cited complications together. Pathophysiologically, blood from aSAH causes occlusion of the arachnoid granulations that is further exacerbated by subsequent meningitis, leading to arachnoid granulation fibrosis and CSF outflow reduction. ${ }^{19}$ Additionally, many forms of meningitis elevate CSF protein levels, which reduces CSF outflow. ${ }^{20}$ The pathophysiology by which pneumonia is associated with shunt dependency is unknown. ${ }^{2,3,20}$ This correlation may be confounded by increased incidence of chest infections in patients with poorer neurological grade, which predisposes them to shunt dependency. ${ }^{20}$ Evidence supporting ische- mic stroke and cerebral vasospasm as shunt-dependency risk factors after aSAH is mixed because of differing definitions of vasospasm and may also be confounded by high Fisher grades. In all but 1 of the included studies, a clinical definition was used in which patients demonstrated symptoms. ${ }^{11,12,15,24}$ One study diagnosed vasospasm on the basis of angiography. ${ }^{19}$ Vasospasm significantly increased shunt dependency only after univariate analysis in 2 studies $^{11,21}$ and was not significant in 3 others. ${ }^{15,19,24}$ Vasospasm was significant after multivariate analysis of 385 patients in 1 study. If ischemic stroke or vasospasm is a risk factor for shunt dependency, then it may result from ischemiainduced downregulation of CSF clearance mechanisms. ${ }^{11}$

Intraventricular blood is a strongly supported risk factor for shunt dependency that was significant in all included studies. This risk is not independent of high Fisher grade, because Fisher grade 4 is assigned to patients with intraventricular blood. Intraventricular blood may contribute to shunt dependency in several ways. First, blood increases CSF viscosity. ${ }^{25}$ Second, blood in the ventricles may cause EVD obstruction, often requiring conversion to a ventriculoperitoneal shunt. Third, blood in the ventricles flows distally and obstructs the arachnoid granulations. ${ }^{24}$

A high Hunt and Hess Scale score was a risk factor with substantial supporting data in our meta-analysis. Because only $1^{9}$ of 11 studies $2,7,9,11-13,15,19,22,28,29$ included in the meta-

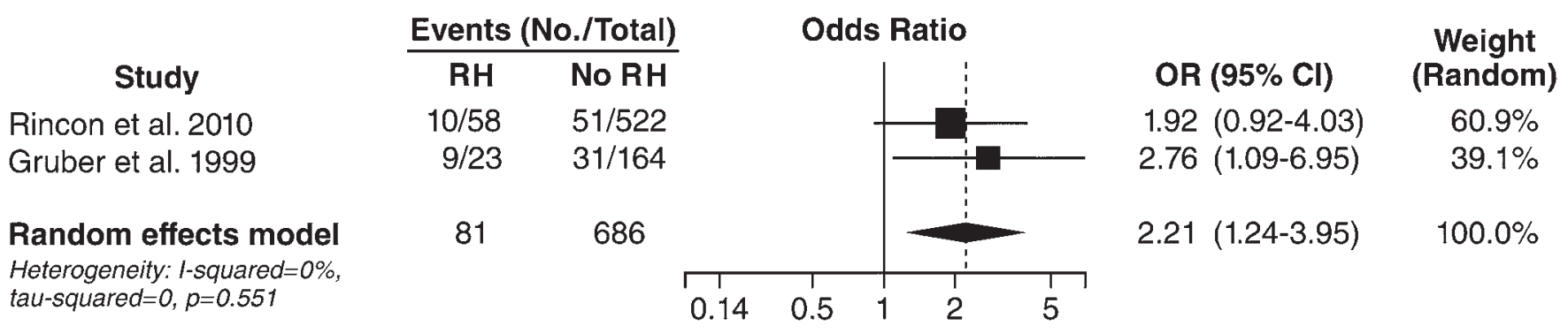

FIG. 7. Forest plot for rehemorrhage $(\mathrm{RH})$ detected on brain imaging as a risk factor for shunt dependency compared with patients without rehemorrhage. 


\begin{tabular}{|c|c|c|c|c|c|}
\hline \multirow[b]{2}{*}{ Study } & \multicolumn{2}{|c|}{ Events (No./lotal) } & \multicolumn{2}{|l|}{ Udos Ratio } & \multirow{2}{*}{$\begin{array}{c}\text { Weight } \\
\text { (Random) }\end{array}$} \\
\hline & PC & No PC & & OR (95\% Cl) & \\
\hline Yu et al. 2014 & $10 / 27$ & $30 / 175$ & & $2.84(1.19-6.82)$ & $7.1 \%$ \\
\hline Lai and Morgan 2013 & $131 / 2019$ & $570 / 8788$ & & $1.00(0.82-1.22)$ & $14.9 \%$ \\
\hline Woernle et al. 2013 & $10 / 39$ & $81 / 350$ & & $1.15(0.54-2.45)$ & $8.2 \%$ \\
\hline Yang et al. 2013 & $2 / 6$ & 20/82 & & $1.55(0.26-9.11)$ & $2.6 \%$ \\
\hline Yamada et al. 2012 & $5 / 15$ & 29/105 & & $1.31(0.41-4.16)$ & $5.0 \%$ \\
\hline O'Kelly et al. 2009 & $87 / 320$ & $498 / 2800$ & & $1.73(1.32-2.25)$ & $14.2 \%$ \\
\hline Chan et al. 2009 & $50 / 65$ & $11 / 24$ & & $3.94(1.47-10.59)$ & $6.1 \%$ \\
\hline Kwon et al. 2008 & $4 / 28$ & $47 / 560$ & & $1.82(0.61-5.46)$ & $5.4 \%$ \\
\hline Dorai et al. 2003 & $61 / 228$ & $92 / 490$ & & $1.58(1.09-2.29)$ & $13.0 \%$ \\
\hline Tapaninaho et al. 1993 & $16 / 57$ & $65 / 778$ & & $4.28(2.28-8.05)$ & $9.6 \%$ \\
\hline Graff-Radford et al. 1989 & $64 / 266$ & $402 / 3255$ & & $2.25(1.67-3.03)$ & $13.8 \%$ \\
\hline Random effects model & 3070 & 17,407 & & $1.85(1.35-2.53)$ & $100.0 \%$ \\
\hline $\begin{array}{l}\text { Heterogeneity: I-squared }=76 \% \text {, } \\
\text { tau-squared }=0.1614, p<0.001\end{array}$ & & 0.09 & 0.5 & & \\
\hline
\end{tabular}

FIG. 8. Forest plot for posterior circulation aneurysm location (PC) as a risk factor for shunt dependency compared with patients with anterior circulation aneurysms (No PC). Posterior circulation aneurysms included those arising from the vertebral, basilar, or posterior communicating arteries or their branches. Anterior circulation aneurysms included those located in all other cerebral blood vessels.

analysis indicated no significance to this risk factor, Hunt and Hess Scale scores are likely useful for predicting shunt dependency. This grading scale is simple to use and widespread.

Our results demonstrated a significant increase in shunt dependency in patients experiencing rehemorrhage after the initial episode of aSAH. The inflammation, clotting, and fibrosis of the arachnoid granulations after the initial ictus leave a smaller reserve for normal CSF drainage, which may be overwhelmed by rehemorrhage. However, data are sparse and mixed. Our analysis was based on only 2 Level B studies, which included a total of 767 patients. The larger study (580 patients) did not show a significant increase in shunt dependency in rehemorrhage patients, ${ }^{19}$ whereas the smaller study (187 patients) did.${ }^{13}$ Further evidence is required to increase confidence in the effects of rehemorrhage on shunt dependency.

Although our analysis showed a statistically significant increase in shunt dependency in patients with posterior circulation aneurysms, this risk factor is likely not currently clinically relevant. First, most early (publication year 1993 or earlier) studies showed highly significant increases in shunt dependency, ${ }^{12,21}$ whereas, with 1 exception, ${ }^{26}$ recent studies found a weak association or no significant risk at all. $311,16,18,26-28$ Second, the division between anterior and posterior circulation may not be specific enough to predict shunt dependency. It may be more useful to evaluate aneurysm location according to individual arteries. Level $B$ evidence shows that basilar artery, vertebral artery, and posterior communicating artery aneurysms increase shunt dependency, especially if the aneurysm is located distally. $3,11,13,16,18,29$ Third, the posterior communicating artery is not traditionally considered part of the posterior circulation. However, many studies in this meta-analysis classified it as part of the posterior circulation. Nevertheless, there may be a pathophysiological link between posterior circulation aneurysms and shunt dependency. Posterior vessels bleed into the high-volume basal cisterns relative to other areas such as the sylvian fissure. ${ }^{11,16,29}$ More subarachnoid blood is associated with higher shunt-dependency rates. ${ }^{11}$ Additionally, posterior aneurysms are more likely than anterior aneurysms to cause intraventricular hemorrhage, which is associated with shunt dependency.11,29

Older age was associated with shunt dependency. Although only 2 studies ${ }^{12,15}$ were included in our meta-analysis, this risk factor was seen in several other studies, ${ }^{11,18}$, $24,27,29$ and both studies in the meta-analysis involved large sample sizes. Only 2 older studies failed to demonstrate increased shunt dependency in the elderly. ${ }^{20,22}$ One proposed reason for the higher prevalence of shunt dependency in

\begin{tabular}{|c|c|c|c|c|c|}
\hline \multirow[b]{2}{*}{ Study } & \multicolumn{2}{|c|}{ Events (No./Total) } & Odds Ratio & \multirow[b]{2}{*}{ OR $(95 \% \mathrm{Cl})$} & \multirow{2}{*}{$\begin{array}{l}\text { Weight } \\
\text { (Random) }\end{array}$} \\
\hline & Age $\geq 60$ & Age $<60$ & & & \\
\hline Kwon et al. 2008 & $33 / 227$ & $33 / 508$ & & $2.45(1.47-4.08)$ & $12.1 \%$ \\
\hline Graff-Radford et al. 1989 & $166 / 908$ & $300 / 2613$ & & $1.72(1.40-2.12)$ & $87.9 \%$ \\
\hline $\begin{array}{l}\text { Fixed effects model } \\
\text { Heterogeneity: } \mathrm{I} \text {-squared }=35.6 \% \text {, }\end{array}$ & 1135 & 3121 & & $1.81(1.50-2.19)$ & $100.0 \%$ \\
\hline (5) & & 0.25 & .5 & & \\
\hline
\end{tabular}

FIG. 9. Forest plot of age $\geq 60$ years as a risk factor for shunt dependency compared with younger patients (<60 years). 


$\quad$ Study
Yu et al. 2014
Yang et al. 2013
Woernle et al. 2013
Brandner et al. 2012
Rincon et al. 2010
Chan et al. 2009
Kwon et al. 2008
de Oliveira et al. 2007
Dorai et al. 2003
Sheehan et al. 1999
Vermeij et al. 1994
Tapaninaho et al. 1993
Random effects model
Heterogeneity: 1 -squared=82.4\%,
tau-squared $=0.3465, p<0.0001$

\begin{tabular}{|c|c|c|}
\hline Events ( & Jo./Total) & Odds Ratio \\
\hline Female & Male & \\
\hline $19 / 122$ & $21 / 81$ & \\
\hline $15 / 63$ & $7 / 25$ & \\
\hline $66 / 257$ & $25 / 132$ & \\
\hline $17 / 46$ & $8 / 22$ & \\
\hline $43 / 391$ & $18 / 189$ & \\
\hline $36 / 57$ & $11 / 32$ & \\
\hline $48 / 498$ & $18 / 239$ & \\
\hline $42 / 241$ & $29 / 144$ & \\
\hline $114 / 479$ & $28 / 236$ & \\
\hline $125 / 445$ & $106 / 211$ & \\
\hline $91 / 398$ & $52 / 262$ & \\
\hline $47 / 397$ & $34 / 438$ & \\
\hline 3394 & 2011 & \\
\hline & 0.12 & 0.5 \\
\hline
\end{tabular}

$\begin{array}{cc}\text { OR (95\% Cl) } & \begin{array}{c}\text { Weight } \\ \text { (Random) }\end{array} \\ 0.53(0.26-1.06) & 7.9 \% \\ 0.80(0.28-2.29) & 5.9 \% \\ 1.48(0.88-2.48) & 9.0 \% \\ 1.03(0.36-2.95) & 5.9 \% \\ 1.17(0.66-2.10) & 8.6 \% \\ 3.27(1.32-8.10) & 6.7 \% \\ 1.29(0.73-2.27) & 8.7 \% \\ 0.84(0.49-1.42) & 8.9 \% \\ 2.35(1.51-3.68) & 9.4 \% \\ 0.39(0.28-0.54) & 9.9 \% \\ 1.20(0.82-1.76) & 9.7 \% \\ 1.60(1.00-2.54) & 9.3 \%\end{array}$

FIG. 10. Forest plot for female sex as a risk factor for shunt dependency compared with male aSAH patients.

older adults is that older individuals often have more comorbidities than younger adults, and pulmonary disease, pneumonia, and hypertension increase shunt dependency in aSAH patients. ${ }^{20,21}$ Also, older adults are more susceptible to brain ischemia and ventricular system damage, ${ }^{29}$ both of which increase shunt dependency.

We have no reason to suspect that female patients are at higher risk of shunt dependency than male patients, as previously suggested. ${ }^{3,11}$ Even studies that reported this risk factor provide no explanation for it. ${ }^{3}$ One study even found that females were significantly protected from shunt dependency (OR 0.39, 95\% CI 0.28-0.54). ${ }^{20}$ The variance in the literature likely represents subtle population differences.

Our study has several limitations. First, several risk factors were evaluated by few studies: hyperglycemia, moderate Glasgow Coma Scale scores, less common neurological grading systems, mechanical ventilation requirement, and hypertension. Consequently, these factors could not be assessed by meta-analysis. Second, evidence for some risk factors, such as female sex, is contradictory, with our meta-analysis indicating no significant risk after combining data. Third, many risk factors (such as intraventricular hemorrhage and high Fisher grade) are not mutually exclusive and may cause overestimation of shunt-dependency risk. This study was not designed to provide independent risk factors for shunt dependency after aSAH. Fourth, our analysis is based on observational studies from a variety of institutions and time periods. Consequently, there is marked heterogeneity among risk factors. We attempted to account for heterogeneity using a random effects model for meta-analysis when the Cochran Q statistic was significant. However, even this may not account for all heterogeneity. Finally, some of our results may be subject to publication bias, although our publication bias analysis (Supplemental Online Data only) indicated no bias in risk factors, except intraventricular blood and high Fisher grade. However, these 2 factors were strongly supported by available evidence.
The benefits of our study are 3 -fold. First, we conducted a thorough literature review, allowing physicians to confidently predict shunt-dependency risk in aSAH patients. Second, all but 3 of the risk factors included are immediately evaluable on presentation by history, physical examination, and imaging. Using these factors to predict risk will not add cost to current standards of care. Third, by organizing published data regarding shunt-dependency risk factors, we have highlighted gaps in our knowledge, which can be used to guide future investigations.

\section{Conclusions}

The most significant risk factor for shunt-dependent hydrocephalus after aSAH was high Fisher grade, followed, in order, by acute hydrocephalus, in-hospital complications, intraventricular blood, high Hunt and Hess Scale score, rehemorrhage, posterior circulation aneurysm location, and age $\geq 60$ years; patient sex was not a significant risk factor.

\section{References}

1. Atkins D, Best D, Briss PA, Eccles M, Falck-Ytter Y, Flottorp $\mathrm{S}$, et al: Grading quality of evidence and strength of recommendations. BMJ 328:1490, 2004

2. Brandner S, Xu Y, Schmidt C, Emtmann I, Buchfelder M, Kleindienst A: Shunt-dependent hydrocephalus following subarachnoid hemorrhage correlates with increased S100B levels in cerebrospinal fluid and serum. Acta Neurochir Suppl 114:217-220, 2012

3. Chan M, Alaraj A, Calderon M, Herrera SR, Gao W, Ruland $\mathrm{S}$, et al: Prediction of ventriculoperitoneal shunt dependency in patients with aneurysmal subarachnoid hemorrhage. J Neurosurg 110:44-49, 2009

4. Cochran WG: The comparison of percentages in matched samples. Biometrika 37:256-266, 1950

5. Cochran WG: Some methods for strengthening the common $\chi^{2}$ tests. Biometrics 10:417-451, 1954

6. Connolly ES Jr, Rabinstein AA, Carhuapoma JR, Derdeyn 
CP, Dion J, Higashida RT, et al: Guidelines for the management of aneurysmal subarachnoid hemorrhage: a guideline for healthcare professionals from the American Heart Association/American Stroke Association. Stroke 43:1711-1737, 2012

7. de Oliveira JG, Beck J, Setzer M, Gerlach R, Vatter H, Seifert $\mathrm{V}$, et al: Risk of shunt-dependent hydrocephalus after occlusion of ruptured intracranial aneurysms by surgical clipping or endovascular coiling: a single-institution series and metaanalysis. Neurosurgery 61:924-934, 2007

8. Dehdashti AR, Rilliet B, Rufenacht DA, de Tribolet N: Shunt-dependent hydrocephalus after rupture of intracranial aneurysms: a prospective study of the influence of treatment modality. J Neurosurg 101:402-407, 2004

9. Demirgil BT, Tugcu B, Postalci L, Guclu G, Dalgic A, Oral $\mathrm{Z}$ : Factors leading to hydrocephalus after aneurysmal subarachnoid hemorrhage. Minim Invasive Neurosurg 46:344348,2003

10. DerSimonian R, Laird N: Meta-analysis in clinical trials. Control Clin Trials 7:177-188, 1986

11. Dorai Z, Hynan LS, Kopitnik TA, Samson D: Factors related to hydrocephalus after aneurysmal subarachnoid hemorrhage. Neurosurgery 52:763-771, 2003

12. Graff-Radford NR, Torner J, Adams HP Jr, Kassell NF: Factors associated with hydrocephalus after subarachnoid hemorrhage. A report of the Cooperative Aneurysm Study. Arch Neurol 46:744-752, 1989

13. Gruber A, Reinprecht A, Bavinzski G, Czech T, Richling B: Chronic shunt-dependent hydrocephalus after early surgical and early endovascular treatment of ruptured intracranial aneurysms. Neurosurgery 44:503-512, 1999

14. Hasan D, Vermeulen M, Wijdicks EF, Hijdra A, van Gijn $\mathrm{J}$ : Management problems in acute hydrocephalus after subarachnoid hemorrhage. Stroke 20:747-753, 1989

15. Kwon JH, Sung SK, Song YJ, Choi HJ, Huh JT, Kim HD: Predisposing factors related to shunt-dependent chronic hydrocephalus after aneurysmal subarachnoid hemorrhage. J Korean Neurosurg Soc 43:177-181, 2008

16. Lai L, Morgan MK: Predictors of in-hospital shunt-dependent hydrocephalus following rupture of cerebral aneurysms. J Clin Neurosci 20:1134-1138, 2013

17. Lewis A, Taylor Kimberly W: Prediction of ventriculoperitoneal shunt placement based on type of failure during external ventricular drain wean. Clin Neurol Neurosurg 125:109_ 113,2014

18. O'Kelly CJ, Kulkarni AV, Austin PC, Urbach D, Wallace MC: Shunt-dependent hydrocephalus after aneurysmal subarachnoid hemorrhage: incidence, predictors, and revision rates. Clinical article. J Neurosurg 111:1029-1035, 2009

19. Rincon F, Gordon E, Starke RM, Buitrago MM, Fernandez A, Schmidt JM, et al: Predictors of long-term shunt-dependent hydrocephalus after aneurysmal subarachnoid hemorrhage. Clinical article. J Neurosurg 113:774-780, 2010

20. Sheehan JP, Polin RS, Sheehan JM, Baskaya MK, Kassell NF: Factors associated with hydrocephalus after aneurysmal subarachnoid hemorrhage. Neurosurgery 45:1120-1128, 1999

21. Tapaninaho A, Hernesniemi J, Vapalahti M, Niskanen M, Kari A, Luukkonen M, et al: Shunt-dependent hydrocephalus after subarachnoid haemorrhage and aneurysm surgery: timing of surgery is not a risk factor. Acta Neurochir (Wien) 123:118-124, 1993
22. Vale FL, Bradley EL, Fisher WS III: The relationship of subarachnoid hemorrhage and the need for postoperative shunting. J Neurosurg 86:462-466, 1997

23. van Gijn J, Hijdra A, Wijdicks EF, Vermeulen M, van Crevel $\mathrm{H}$ : Acute hydrocephalus after aneurysmal subarachnoid hemorrhage. J Neurosurg 63:355-362, 1985

24. Vermeij FH, Hasan D, Vermeulen M, Tanghe HL, van Gijn J: Predictive factors for deterioration from hydrocephalus after subarachnoid hemorrhage. Neurology 44:1851-1855, 1994

25. Wang YM, Lin YJ, Chuang MJ, Lee TH, Tsai NW, Cheng $\mathrm{BC}$, et al: Predictors and outcomes of shunt-dependent hydrocephalus in patients with aneurysmal sub-arachnoid hemorrhage. BMC Surg 12:12, 2012

26. Woernle CM, Winkler KM, Burkhardt JK, Haile SR, Bellut D, Neidert MC, et al: Hydrocephalus in 389 patients with aneurysm-associated subarachnoid hemorrhage. J Clin Neurosci 20:824-826, 2013

27. Yamada S, Nakase H, Park YS, Nishimura F, Nakagawa I: Discriminant analysis prediction of the need for ventriculoperitoneal shunt after subarachnoid hemorrhage. J Stroke Cerebrovasc Dis 21:493-497, 2012

28. Yang TC, Chang CH, Liu YT, Chen YL, Tu PH, Chen HC: Predictors of shunt-dependent chronic hydrocephalus after aneurysmal subarachnoid haemorrhage. Eur Neurol 69:296303, 2013

29. Yu H, Zhan R, Wen L, Shen J, Fan Z: The relationship between risk factors and prognostic factors in patients with shunt-dependent hydrocephalus after aneurysmal subarachnoid hemorrhage. J Craniofac Surg 25:902-906, 2014

\section{Disclosures}

Dr. Hanel reports being a consultant for Covidien, Codman, Stryker, and MicroVention; owning direct stock in Blockade; and serving on the scientific advisory board for Medina.

\section{Author Contributions}

Conception and design: Wilson, Safavi-Abbasi. Acquisition of data: Wilson, Safavi-Abbasi. Analysis and interpretation of data: Wilson, Safavi-Abbasi, Zhao. Drafting the article: Wilson, Safavi-Abbasi. Critically revising the article: Spetzler, Wilson, Safavi-Abbasi, Sun, Kalani, Levitt, Hanel, Sauvageau, Mapstone, Albuquerque, McDougall, Nakaji. Reviewed submitted version of manuscript: Spetzler, Wilson, Safavi-Abbasi. Statistical analysis: Zhao. Administrative/technical/material support: Spetzler, Wilson, Safavi-Abbasi. Study supervision: Spetzler, Safavi-Abbasi.

\section{Supplemental Information Online-Only Content}

Supplemental material is available with the online version of the article.

Supplemental Figures. http://thejns.org/doi/suppl/10.3171/ 2015.11.JNS152094.

\section{Correspondence}

Robert F. Spetzler, c/o Neuroscience Publications, Barrow Neurological Institute, St. Joseph's Hospital and Medical Center, 350 W Thomas Rd., Phoenix, AZ 85013.email: neuropub@ dignityhealth.org. 